\title{
Régulation médicale : mise au point 2018 et développements futurs
}

\author{
Emergency Medical Dispatch: State of the Art in 2018 and Future Developments
}

\section{F. Dami · V. Fuchs $\cdot$ V. Berthoz $\cdot$ P.-N. Carron}

Reçu le 9 mai 2018; accepté le 2 septembre 2018

(C) SFMU et Lavoisier SAS 2018

Résumé En 2018, les centrales de régulation d'urgences médicales devraient toutes être en mesure d'offrir certaines plus-values essentielles aux patients : une réanimation par téléphone avec accompagnement des témoins jusqu'à l'arrivée des secours, l'utilisation de réseaux de premiers répondants, ainsi que les défibrillateurs automatiques des domaines privés et publics via des applications Internet, l'assistance aux témoins pour réaliser des injections d'épinéphrine lors de réactions allergiques sévères ou de chlorhydrate de glucagon lors d'hypoglycémie. En complément, le bénéfice attendu des centrales d'urgences médicales visà-vis du système de soins dans son ensemble implique une meilleure efficience quant à l'engagement des moyens dont elles disposent. Cela ne peut se faire qu'en collectant les données liées à l'engagement des moyens (type, nombre, niveau de priorité) et en les corrélant avec la gravité de la situation observée sur site par les intervenants. Cette analyse permet d'estimer les taux de sur-triage et de sous-triage de la centrale lors de la décision d'envoi des moyens. L'utilisation appropriée des signaux prioritaires qui en découle permet également de réduire les risques des intervenants et de la population générale. Finalement, les fusions de centrales permettent de rationaliser certains coûts fixes, de faire face à des difficultés de recrutement et de formation, mais également d'augmenter la qualité de la régulation offerte à la population.

Mots clés Régulation · Efficience · Réanimation · Sur-triage $\cdot$ Sous-triage

F. Dami $(\bowtie) \cdot$ P.-N. Carron

Service des urgences, université de Lausanne, centre hospitalier universitaire Vaudois (CHUV), Bugnon 46, CH-1011 Lausanne, Lausanne, Suisse e-mail : fabrice.dami@chuv.ch

F. Dami - V. Fuchs $\cdot$ V. Berthoz

Fondation Urgences-Santé,

régulation médicale cantons de Vaud et Neuchâtel,

Rue du Dr César-Roux 29, CH-1005 Lausanne, Suisse
Abstract In 2018, all dispatch centres should offer addedvalue services to the patients, such as telephone cardiopulmonary resuscitation (CPR), use of first responders and public automatic external defibrillator (AED) through Apps, coaching witnesses to inject epinephrine in case of anaphylaxis or glucagon when facing a patient with hypoglycemia. Dispatch centres must also contribute to a better use of available resources (ambulances, prehospital physicians). This can only be achieved by collecting data based on level of priority used, and comparing it to the severity of cases described by paramedics once on site. This may allow to measure over and under triage by dispatch centres. The appropriate use of lights and sirens may also contribute to reduce risks for emergency medical services (EMS) personal, patients and the general population. Finally, mergers of dispatch centres may reduce fixed costs, ease recruitment and continuous education issues, and improve the quality of care.

Keywords Dispatch · Efficiency · Telephone CPR · Under-triage $\cdot$ Over-triage

\section{Introduction}

Pendant des années, la régulation médicale d'urgence ${ }^{1}$ à travers le monde s'est limitée à envoyer rapidement les moyens les mieux adaptés à la bonne adresse. Il existe aujourd'hui un large consensus pour affirmer que cette réponse n'est plus suffisante. La régulation doit non seulement apporter une réponse précoce en garantissant une plus-value pour le patient (qualité), mais elle doit également soutenir le système de soins dans son ensemble (efficience).

L'objectif de cette mise au point est de décrire quels sont les bénéfices attendus des centrales de régulation d'urgences médicales (ci-après « centrales ») en 2018, que ce soit

\footnotetext{
${ }^{1}$ Nous n'évoquerons ici que la régulation urgente, soit la gestion des urgences vitales supposées. La médecine de garde ne sera pas abordée.
} 
pour les patients comme pour le système de soins dans son ensemble.

Le terme " régulateur 》 dans le présent manuscrit s'adresse indifféremment à toute personne qui réalise l'anamnèse, décide de l'engagement de moyens et propose des conseils par téléphone. À l'exception de la France qui a décidé de confier cette mission majoritairement à des médecins, les autres pays utilisent des profils professionnels différents (infirmier, ambulancier, assistant en régulation médicale sans expérience dans les soins médicaux). Cette mise au point n'abordera pas les avantages et les inconvénients des différents systèmes de régulation existants.

\section{Plus-value pour le patient}

\section{Réanimation par téléphone}

Lorsque l'on parle de plus-value pour le patient en lien avec la régulation médicale urgente, la réanimation par téléphone vient souvent en premier à l'esprit [1]. La survie après un arrêt cardiorespiratoire (ACR) dépend largement de l'initiation immédiate d'une réanimation cardiopulmonaire par les témoins. Il est ainsi clairement démontré que plus la proportion de patients en ACR bénéficiant d'un massage cardiaque par les témoins est élevée, meilleure est la survie globale dans cette communauté. En favorisant le massage cardiaque par les témoins, les centrales améliorent ainsi le taux de survie des ACR [1]. Malheureusement, le taux de centrales qui proposent ces gestes de secours est encore trop faible : $50 \%$ des centrales aux États-Unis en 2012 [2] et $81 \%$ en Europe en 2013 dans le cadre d'une enquête non publiée [3]. Audelà de l'implémentation systématique de ce type de stratégie, l'amélioration de la qualité des réanimations guidées par téléphone constitue également un enjeu qui passe par la formation des régulateurs, quelle que soit leur formation de base. Ils doivent être sensibilisés au bénéfice de ces conseils, entraînés à reconnaître les signes d'un ACR au téléphone tels que l'inconscience, une respiration anormale, absente ou agonique ainsi que les convulsions. En effet, $2 \%$ des appels pour convulsions non traumatiques chez l'adulte représentent un premier signe d'ACR (bas débit cérébral), raison pour laquelle certains centres rappellent systématiquement deux minutes après l'appel initial les témoins se trouvant auprès de patients présentant des convulsions pour s'assurer de la qualité de la respiration. Cela permet d'évaluer la nécessité de débuter des manœuvres de réanimation [4]. Si les régulateurs doivent être capables d'initier une réanimation guidée par téléphone, ils doivent également et surtout rester en ligne pendant toute la durée de la réanimation jusqu'à l'arrivée des secours (pour autant que l'activité de la centrale le permette, les appels entrants demeurant prioritaires). Ils doivent pouvoir s'appuyer en tout temps sur une procédure écrite, la description de ces gestes au téléphone nécessitant l'utilisation de mots simples et adaptés à l'attention des témoins. Ils doivent également disposer sur leur poste de travail d'un métronome (par exemple via une application Web gratuite) qui leur donne la fréquence recommandée pour effectuer le massage cardiaque. Il est en effet difficile, même pour des experts, de spontanément trouver la juste fréquence. Cette stratégie, impliquant de rester en ligne le plus longtemps possible avec le témoin, est appelée « réanimation par téléphone de haute performance » [5] et s'oppose à celle des centrales qui proposent uniquement aux témoins de débuter la réanimation sans autre forme de soutien. En sus de la reconnaissance de la respiration agonique qui est classiquement le point décrit comme étant le plus compliqué à identifier par les régulateurs [6], il est également indispensable de les rassurer quant à l'absence de conséquences en cas de massage cardiaque prodigué à une personne qui ne serait pas en ACR. Le bénéfice potentiel de faire masser les témoins au moindre doute surpasse ainsi largement les rares effets secondaires d'un massage inopportun. La centrale de King County à Seattle, pionnière en matière de réanimation par téléphone, propose même l'adage «no-no-go »: « not conscious » (inconscience), « no breathing » (absence de respiration), «go resuscitation » (débuter la réanimation). Cette séquence permet d'aller rapidement à l'essentiel, de proposer une réanimation au moindre doute, de ne pas perdre de temps et surtout de réduire au maximum le risque de ne pas proposer de massage cardiaque à un patient qui en aurait besoin.

Il est essentiel pour les centrales de mesurer ce type d'activité, via des indicateurs reconnus. Le rôle des responsables de la centrale est de mesurer la performance de leur équipe en monitorant le taux de reconnaissance des ACR, le temps nécessaire pour reconnaitre l'ACR et le temps nécessaire pour faire débuter la réanimation par le témoin. Ces indicateurs permettent également de se comparer aux résultats déjà publiés et d'améliorer la qualité de leurs réponses $[7,8]$.

\section{Localisation des défibrillateurs et premiers répondants}

Les centrales doivent pouvoir disposer d'outils permettant la géolocalisation précise des défibrillateurs externes automatiques (DEA) dans les domaines public et privé, tout en ayant la possibilité de mobiliser des premiers répondants (professionnels ou civils) disposant d'une formation de base en réanimation. Ces mesures sont complémentaires à la réanimation par téléphone et ont démontré un impact favorable sur la survie en permettant de raccourcir la période de no-flow ainsi que les délais avant la défibrillation $[9,10]$. La figure 1 résume ce qui peut être appelé « la fenêtre du régulateur », soit toute la période pendant laquelle ce dernier, avec l'aide du témoin et des moyens dont il dispose, peut influer sur le pronostic du patient avant même l'arrivée des secours sur site [11,12]. 
La figure 2 montre une nouvelle manière d'illustrer la chaîne des secours en mettant en avant, pour la toute première fois, le rôle fondamental de la régulation lors de la gestion des appels en cas d'ACR [13]. Il s'agit là d'un signal fort, encourageant la reconnaissance d'une activité trop souvent méconnue et sous-estimée.

\section{Gestes de premier secours à distance}

Si la réanimation des ACR guidée par téléphone demeure le geste de secours le plus fréquemment décrit dans la littérature, d'autres gestes doivent également pouvoir être proposés par le régulateur en attendant l'arrivée des secours : allonger le patient et comprimer un point de saignement, voire poser un garrot lors d'une hémorragie, mettre le patient en position latérale de sécurité lors d'un trouble de la conscience, sécurisation des lieux et mise à l'abri des témoins lors d'un accident, ouverture des fenêtres et évacuation des lieux lors d'une suspicion d'intoxication au monoxyde de carbone, réalisation en cas d'asphyxie causée par la présence d'un corps étranger dans les voies aériennes des manœuvres de Heimlich (adulte) ou de Mofenson (enfant).

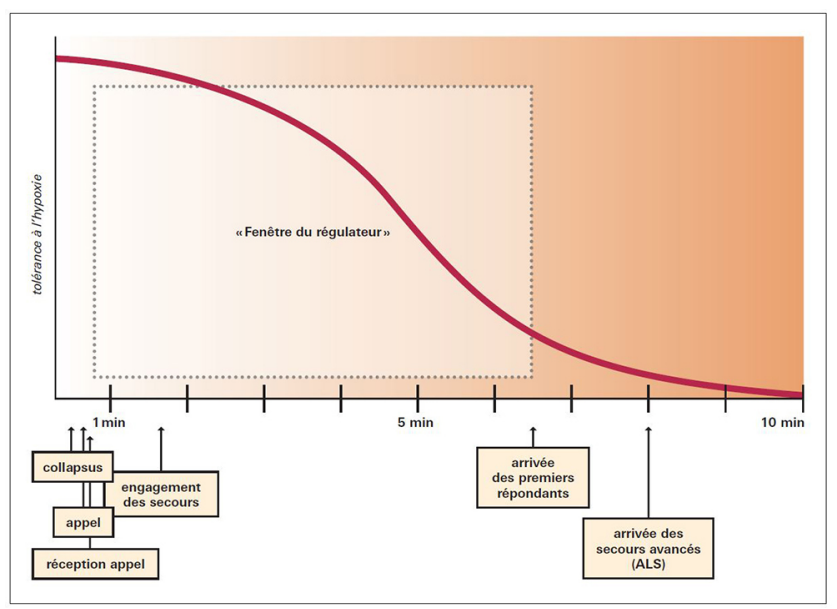

Fig. 1 Fenêtre du régulateur - exemple chronologique d'un appel pour un arrêt cardiaque, selon Carron et al. Et adapté de Breckwoldt et al. $[11,12]$ (reproduit avec autorisation)

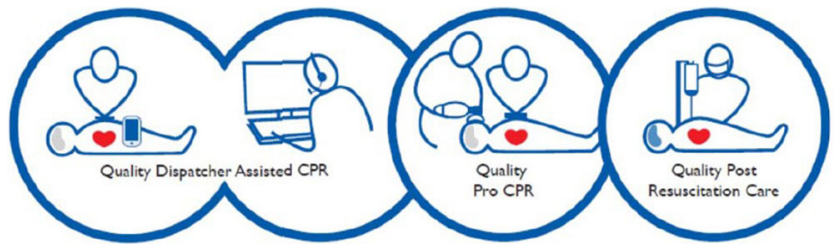

Fig. 2 La chaîne des secours lors d'un arrêt cardiaque, selon Bobrow et al. [13] (reproduit avec autorisation)

\section{Traitements médicamenteux à distance}

Dans certaines situations, le patient nécessite de recevoir rapidement un traitement médicamenteux. Les régulateurs doivent être capables de proposer l'administration de ces traitements, ce qui signifie qu'ils doivent être formés non seulement à reconnaître les symptômes et éléments de gravité posant l'indication au traitement, mais également à enseigner par téléphone la prise de ces traitements. L'American Heart Association (AHA) a par exemple validé la prise d'acide acétylsalicylique (160-325 mg) per os par le patient sur préavis du régulateur lors de douleurs thoraciques suspectes de syndrome coronarien aigu [14]. L'injection d'épinéphrine à l'aide de stylos injectables par le patient lui-même ou par des témoins, guidés par les régulateurs, permet de gagner de précieuses minutes dans le traitement des suspicions d'anaphylaxie [15]. Par analogie, les hypoglycémies sont des motifs d'appels fréquents, et de nombreux patients diabétiques disposent chez eux d'une dose de chlorhydrate de glucagon injectable. Les régulateurs doivent y penser et également guider le témoin à réaliser cette injection lorsque le patient n'est plus capable de prendre du glucose par lui-même.

Enfin, même si nous ne sommes pas confrontés en Europe à une épidémie d'overdoses aux opiacés comme le traversent en ce moment les États-Unis d'Amérique, certaines communautés à risque bénéficient de la distribution de dispositifs d'injection intranasal de naloxone. Pour ces situations, il est nécessaire que les régulateurs soient formés à reconnaître les signes d'overdose et soient capables de guider les témoins pour traiter le patient.

\section{Plus-value pour le système de soins}

\section{Sur-triage et sous-triage}

Ces notions universelles reposent sur des principes simples du point de vue de la centrale : le sur-triage représente un engagement de moyens superflus par rapport à la situation observée par les intervenants sur site. Le sous-triage représente à l'inverse un engagement de moyens insuffisants par rapport à la situation observée sur site. Les définitions opérationnelles précises des sur-triage et sous-triage vont nécessairement être propres à chaque système de soins ou à chaque centrale, en fonction des moyens à disposition (nombre de vecteurs de transport, type de moyens, nombre de médicalisations) et des compétences des intervenants, en particulier non médicaux (sapeurs-pompiers, infirmiers, techniciens ambulanciers, ambulanciers diplômés, voire paramedics). Ces notions de sur- et de sous-triage par les centrales bénéficient d'un intérêt récent dans la littérature, les moyens dont les centrales disposent étant par définition limités. 
Il est ainsi de la responsabilité des régulateurs, en appliquant les procédures internes à leur centrale, d'engager de manière efficiente les moyens dont ils disposent tant quantitativement que qualitativement (ambulance simple, équipe secouriste, équipe médicale préhospitalière, hélicoptère médicalisé ou non). Les moyens engagés doivent être adaptés à l'urgence médicale suspectée, en termes de nombre, de matériel et de compétences. Pour mesurer cette performance, les centrales doivent collecter de manière systématique des indicateurs permettant de documenter les niveaux (priorités) des engagements effectués, en fonction des symptômes ou de la gravité supposée, et de les corréler ensuite à un score de gravité relevé par les intervenants préhospitaliers sur site [16]. Ce type d'analyse permet de modifier les règles d'engagement et, par exemple, de ne plus envoyer systématiquement des moyens en priorité absolue pour des situations qui relèveraient d'une faible gravité sur site (exemples : douleurs thoraciques atypiques, dyspnée sans critères de gravité, etc.).

À terme, ces indicateurs de sur-/sous-triage peuvent également être appliqués de manière individuelle à chaque régulateur, permettant de mieux cibler sa formation continue. En l'absence de consensus, par analogie avec les recommandations émises par l'Association des chirurgiens américains (American College of Surgeons) qui définit quel patient traumatisé doit être transporté vers un trauma center, des taux de sur-triage de $25-35 \%$ et de sous-triage de $5 \%$ sont acceptés [17]. Il convient cependant de rappeler que les régulateurs, à l'inverse des intervenants sur site, sont encore aujourd'hui « aveugles » et n'ont que quelques minutes pour définir une priorité d'engagement. Ces éléments expliquent le taux de sur-triage généralement élevé des centrales, pouvant atteindre $75 \%$ dans les rares publications sur le sujet [16].

\section{Utilisation des signaux prioritaires (feux bleus)}

Il est clairement démontré que les accidents surviennent proportionnellement plus fréquemment lorsque les véhicules roulent en utilisant les signaux prioritaires [18]. Lors de ces événements, des occupants du véhicule (professionnels ou patients) de même que des passants peuvent être blessés. Il est également communément admis que seuls 5-7\% des appels reçus par les centrales représentent une urgence vitale. Si ces missions pouvaient être connues à l'avance, le taux d'engagement de véhicules avec signaux prioritaires enclenchés serait probablement du même ordre, c'està-dire de moins de $10 \%$. Il est de la responsabilité des centrales d'utiliser à bon escient ces signaux prioritaires lors de l'envoi des moyens de secours sur site, en utilisant plusieurs niveaux d'engagement ; ceux-ci, corrélés à un score de gravité établi sur site, peuvent être des indicateurs de sur- et de sous-triage (voir ci-dessus). Les centrales ne devraient pas abuser des feux bleus par facilité ; si les régulateurs peuvent penser qu'ils offrent ainsi la meilleure réponse au patient, ils prennent également la responsabilité de la mise en danger injustifiée des intervenants à bord des véhicules ainsi que la population générale. Ce sont par contre les équipes préhospitalières qui décident de l'utilisation des signaux prioritaires lors du transport du patient vers sa destination.

\section{Centralisation des compétences}

En termes de santé publique, la concentration de compétences dans les centrales peut permettre de rationaliser certaines pratiques. Cela se fait quotidiennement lors de suspicions d'ACR : on sollicite des témoins pour débuter la réanimation, qu'ils soient formés ou non aux gestes de base, car on sait que leur action, guidée par les régulateurs, peut dramatiquement modifier le devenir du patient. Ce principe peut également être appliqué à d'autres situations. On l'a vu cidessus, les régulateurs doivent être capables d'identifier les réactions allergiques sévères. Dans les milieux à risque (piscine, centres de loisirs extérieurs), centraliser cette compétence peut permettre d'éviter de former l'ensemble du personnel qui ne serait individuellement que rarement confronté à un tel événement.

\section{Concentration-fusion des centrales}

Les centrales d'urgences sont considérées comme d'indispensables, mais coûteuses composantes des systèmes de soins préhospitaliers (ressources humaines, logiciels de régulation, matériel informatique et de communication). Il n'existe aucune évidence relative à la taille de la population idéale qui devrait être desservie par une centrale [19]. Le nombre de centrales dans une région ou un pays est généralement le fruit d'un contexte historique local, souvent fondé sur des systèmes de santé décentralisés. La technologie actuelle d'informatisation et de géolocalisation, en particulier par GPS (global positioning system), abolit cependant le besoin de proximité entre les centrales et la population qu'elles desservent. Ce principe s'oppose à une vision plus historique, prônant des régulateurs qui connaissent les détails du terrain. Dans les faits cependant, ces derniers ne peuvent pas, même à l'échelle d'un département, connaître chaque village, chaque ruelle. Parallèlement, en raison d'un besoin accru de réduction des coûts, il existe une réelle difficulté à maintenir l'ensemble de ces structures hautement spécialisées. Pour ces raisons, on observe en Europe depuis quelques années une tendance à une réduction du nombre de centrales, en opérant des fusions (Tableau 1).

Ce phénomène de rationalisation est nécessaire non seulement pour contrôler les coûts, mais également pour faire face aux difficultés récurrentes de recrutement du personnel qualifié et du maintien des compétences (formation de base 


\begin{tabular}{|c|c|c|c|c|}
\hline & Année & $\begin{array}{l}\text { Population (Millions } \\
\text { d'habitants) }\end{array}$ & $\begin{array}{l}\text { Nombre de centrales } \\
\text { d'alarme }\end{array}$ & $\begin{array}{l}\text { Population desservie } \\
\text { par centrale (Millions } \\
\text { d'habitants) }\end{array}$ \\
\hline \multicolumn{5}{|l|}{ Pays } \\
\hline Islande & 2004 & 0,3 & 2 & 0,15 \\
\hline Norvège & 2004 & 4,5 & 22 & 0,20 \\
\hline Allemagne & 2006 & 82,5 & 320 & 0,25 \\
\hline Suisse & 2006 & 7,5 & 22 & 0,34 \\
\hline Finlande & 2004 & 5,3 & 15 & 0,35 \\
\hline Suède & 2004 & 8,9 & 20 & 0,45 \\
\hline Suisse & 2015 & 8 & 17 & 0,47 \\
\hline Suède & 2011 & 9,5 & 18 & 0,53 \\
\hline France & 2004 & 60,4 & 105 & 0,57 \\
\hline Danemark & 2004 & 5,4 & 9 & 0,60 \\
\hline Finlande & 2015 & 5,2 & (7, projet) & 0,74 \\
\hline \multicolumn{5}{|l|}{ Villes } \\
\hline Lausanne & 2015 & 0,8 & 1 & 0,8 \\
\hline Fort Myers & 2010 & 0,8 & 1 & 0,8 \\
\hline Montréal & 2011 & 2,3 & 1 & 2,3 \\
\hline Miami & 2010 & 2,5 & 1 & 2,5 \\
\hline Phoenix & 2010 & 4,2 & 1 & 4,2 \\
\hline
\end{tabular}

et continue). Finalement, la population peut bénéficier d'une amélioration de la qualité de la régulation lorsqu'un petit territoire est repris par une plus grande entité qui bénéficie souvent de plus de moyens et d'une plus grande expérience [20].

\section{Avenir en régulation médicale}

\section{Vidéophone}

Une communication vidéo entre l'appelant et le régulateur a déjà été testée avec succès lors de consultations ne relevant pas de l'urgence vitale. La technologie nécessite encore à ce jour quelques manipulations de la part de l'appelant (validation d'un texto par exemple), ce qui n'est pas réalisable lors d'une urgence vitale, notamment en raison du stress. Lorsque la technologie le permettra, la plus-value pour le patient devrait être significative, car le régulateur pourra mieux évaluer son état et donc être plus précis dans sa réponse (choix de la priorité, engagement ou non de la médicalisation quand elle est disponible). Il pourra également mieux guider le patient ou les témoins pour réaliser des gestes de secours (réanimation guidée par téléphone avec feedback vidéo) [21-23] ou proposer des traitements à distance (injection d'épinéphrine par exemple).

\section{Télémédecine}

La télémédecine représente l'échange de données entre l'équipe préhospitalière et les centrales ou les hôpitaux. L'envoi d'électrocardiogrammes 12 dérivations en est l'exemple le plus courant, appliqué dans de nombreux systèmes pour permettre une ouverture précoce de la salle de cathétérisme en cas d'infarctus du myocarde avec surélévation du segment ST (STEMI). Les communications orales depuis le site de l'intervention à destination des équipes hospitalières pour la prise en charge rapide des accidents vasculaires aigus sont également déjà bien implantées. L'échange d'images à l'aide de lunettes connectées ou de caméras en position thoracique est maintenant en voie de développement $[24,25]$. Des limitations techniques existent encore actuellement, principalement en raison de la couverture incomplète du réseau $3 \mathrm{G}$ ou $4 \mathrm{G}$, mais également parfois pour des raisons médicolégales (droit d'acquérir des images, conservation de ces images, aspects médicolégaux). Ce type d'échange devrait néanmoins permettre de mieux guider les équipes sur site, de potentiellement permettre aux équipes préhospitalières non médicalisées de ne pas transporter le patient si ce dernier ne présente pas une situation aiguë, mais également de réduire les besoins en médicalisation préhospitalière sur site.

La télémédecine pourrait par ailleurs permettre de médicaliser plus facilement à distance des missions qui ne le sont actuellement pas, notamment les situations en maison de 
retraite pour des plaintes non traumatiques. Il devrait être possible par cette technologie de traiter sur place certains patients en leur évitant ainsi un déplacement dans des services d'urgences déjà surchargés et souvent peu adaptés aux patients âgés [26]. L'utilisation de la télémédecine dépendra bien évidemment des spécificités de chaque système, des compétences des intervenants sur le terrain et des ressources médicales en centrale et sur site. Dans le cadre de la médecine de proximité, hors urgence vitale, il est également possible d'envisager, pour pallier la pénurie de médecins généralistes, d'envoyer à domicile des infirmières expérimentées en soins d'urgence et de leur offrir un soutien médical à distance.

\section{Intelligence artificielle}

Il est reconnu que la reconnaissance d'un ACR (voir cidessus) ou d'un accident vasculaire cérébral (AVC), en l'absence de soutien par vidéo, est difficile. La sensibilité et spécificité du dépistage de l'AVC en régulation est particulièrement décevante [27,28]. L'intelligence artificielle pourrait à l'avenir aider le régulateur en analysant les mots utilisés par l'appelant (" attaque ", " bouche tordue ", " discours empâté ») et permettrait de faire suspecter la présence d'un AVC [29]. Le projet européen CORTI est en train de faire de même pour le dépistage de l'ACR [30].

\section{Conclusion}

Les centrales doivent être en mesure d'offrir la réanimation par téléphone, une mesure qui a démontré un bénéfice évident pour les patients. Elles devraient également offrir d'autres gestes de secours et de traitements à distance, principalement l'épinéphrine en intramusculaire lors de suspicion d'anaphylaxie.

Les centrales, tout en demeurant centrées sur l'intérêt du patient, doivent promouvoir une utilisation efficiente des moyens de secours qui sont mis à leur disposition, y compris si cela passe par des projets de fusion. Il leur incombe également de suivre les publications relatives à leur domaine de compétence et de proposer de nouvelles procédures afin de faire évoluer la régulation médicosanitaire dans leur région [31].

Conflits d'intérêts : les auteurs déclarent ne pas avoir de conflits d'intérêts.

\section{Références}

1. Lerner EB, Rea TD, Bobrow BJ, et al (2012) Emergency medical service dispatch cardiopulmonary resuscitation prearrival instructions to improve survival from out-of-hospital cardiac arrest. Circulation 125:648-55
2. Sutter J, Panczyk M, Spaite DW, et al (2015) Telephone CPR instructions in emergency dispatch systems: qualitative survey of 911 call centers. West J Emerg Med 16:736-42

3. European Resuscitation Council Dispatch Centre Survey (EDiCeS) on telephone-assisted cardiopulmonary resuscitation. https://www. erc.edu/projects/edices (Dernier accès le 18 mars 2018)

4. Dami F, Rossetti AO, Fuchs V, et al (2012) Proportion of outof-hospital adult non-traumatic cardiac or respiratory arrest among calls for seizure. Emerg Med J 29:758-60

5. Bobrow BJ, Spaite DW, Vadeboncoeur TF, et al (2016) Implementation of a regional telephone cardiopulmonary resuscitation program and outcomes after out-of-hospital cardiac arrest. JAMA Cardiol 1:294-302

6. Fukushima H, Imanishi M, Iwami T, et al (2015) Abnormal breathing of sudden cardiac arrest victims described by laypersons and its association with emergency medical service dispatcher-assisted cardiopulmonary resuscitation instruction. Emerg Med J 32:314-7

7. Lewis M, Stubbs BA, Eisenberg MS, et al (2013) Dispatcherassisted cardiopulmonary resuscitation time to identify cardiac arrest and deliver chest compression instructions. Circulation 128:1522-30

8. Dami F, Heymann E, Pasquier M, et al (2015) Time to identify cardiac arrest and provide dispatch-assisted cardio-pulmonary resuscitation in a criteria-based dispatch system. Resuscitation 97:27-33

9. Ringh M, Rosenqvist M, Hollenberg J, et al (2015) Mobile-phone dispatch of laypersons for CPR in out-of-hospital cardiac arrest. N Engl J Med 372:2316-25

10. Fredman D, Svesson L, Yifang B, et al (2016) Expanding the first link in the chain of survival - experiences from dispatcher referral of callers to AED locations. Resuscitation 107:129-34

11. Dami F, Fuchs V, Deslarzes JP (2013) Régulation sanitaire. In: Carron PN, Dami F, Frei O, et al (eds) Médecine d'urgence préhospitalière. Médecine \& Hygiène Ed, Genève, pp 10-17

12. Breckwoldt J, Schloesser S, Arntz HR (2009) Perceptions of collapse and assessment of cardiac arrest by bystanders of outof-hospital cardiac arrest (OOHCA). Resuscitation 80:1108-13

13. Bobrow B, Panczyk M (2017) 2017 Emergency Cardiovascular Care Update (ECCU) pre-conference workshop. New Orleans

14. American Heart Association (2015) Web-based integrated 2010 \& 2015 American Heart Association guidelines for cardiopulmonary resuscitation and emergency cardiovascular care. Part 9: Acute coronary syndrom. https://eccguidelines.heart.org/index.php/ circulation/cpr-ecc-guidelines-2/part-9-acute-coronary-syndromes (Dernier accès le 3 avril 2018)

15. Gloaguen A, Cesareo E, Vaux J, et al (2016) Prise en charge de l'anaphylaxie en médecine d'urgence. Recommandations de la Société française de médecine d'urgence (SFMU) en partenariat avec la Société française d'allergologie (SFA) et le Groupe francophone de réanimation et d'urgences pédiatriques (GFRUP), et le soutien de la Société pédiatrique de pneumologie et d'allergologie (SP2A). Ann Fr Med Urg 6:342-64

16. Dami F, Golay C, Pasquier M, et al (2015) Prehospital triage accuracy in a criteria based dispatch centre. BMC Emerg Med 15:32

17. American College of Surgeons, Committee on Trauma (2014) Resources for optimal care of the injured patients. https://www.facs. org/ /media/files/quality\%20programs/trauma/vrc\%20resources/ resources $\% 20$ for $\% 20$ optimal $\% 20$ care.ashx (Dernier accès le 3 avril 2018)

18. Kahn CA, Pirrallo RG, Kuhn EM, et al (2009) Characteristics of fatal ambulance crashes in the United States: an 11-year retrospective analysis. Prehosp Emerg Care 5:261-9

19. Dami F, Fuchs V, Hugli O (2015) Dispatch centres: what is the right population catchment size? SJTREM 23:3 
20. Moser A, Mettler A, Fuchs V, et al (2017) Merger of two dispatch centres: does it improve quality and patient safety? SJTREM 25:40

21. You JS, Park S, Chung SP, et al (2008) Performance of cellular phones with video telephony in the use of automated external defibrillators by untrained laypersons. Emerg Med J 25:597-600

22. Melbye S, Hotvedt M, Bolle SR (2014) Mobile videoconferencing for enhanced emergency medical communication - a shot in the dark or a walk in the park? A simulation study. SJTREM 22:35

23. Bolle SR, Johnsen E, Gilbert M (2011) Video calls for dispatcher-assisted cardiopulmonary resuscitation can improve the confidence of lay rescuers - surveys after simulated cardiac arrest. J Telemed Telecare 17:88-92

24. Skorning M, Bergrath S, Rörtgen D, et al (2012) Teleconsultation in pre-hospital emergency medical services: real-time telemedical support in a prospective controlled simulation study. Resuscitation 83:626-32

25. Rörtgen D, Bergrath S, Rossaint R, et al (2012) Comparison of physician staffed emergency teams with paramedic teams assisted by telemedicine - a randomized, controlled simulation study. Resuscitation 84:85-92

26. Boulet MC, Dami F, Hugli O, et al (2015) Seniors aux urgences, une question d'hospitalité ? Rev Med Suisse 11:2338-41

27. Dami F, Emery A, Pasquier M, et al (2017) Proposition and operational characteristics of a new dispatch scale to specifically identify acute strokes. Eur J Emerg Med 24:202-7

28. Berglund A, von Euler M, Schenck-Gustafsson K, et al (2015) Identification of stroke during the emergency call: a descriptive study of callers' presentation of stroke. BMJ Open 5:e007661. doi: 10.1136/bmjopen-2015-007661(Dernier accès le 29 août 2018)

29. Richards CT, Wang B, Markul E, et al (2017) Identifying key words in 911 calls for stroke: a mixed methods approach. Prehosp emeg care 21:761-6

30. European Emergency Number Association (2018) The CORTI project. http://eena.org/download.asp?item id $=267$ (Dernier accès le 6 août 2018)

31. National Association of EMS Physicians (2008) Position paper: emergency medical dispatch. Prehosp Emerg Care 12:217 